\title{
CME Rheumatology (108459): self-assessment questionnaire
}

\author{
Edited by Simon J Bowman and Tahseen A Chowdhury
}

\section{SAQs and answers are ONLINE for RCP fellows and collegiate members}

SAQs and answers are ONLINE for RCP fellows and collegiate members

\section{Format}

Candidates are asked to choose the best answer from the five possible answers. This best of five format is used in many medical examinations, however the questions are not intended to be representative of those used in the MRCP(UK) Part 1 or Part 2 Written Examinations.

\section{The answering process}

1 Go to www.rcplondon.ac.uk/SAQ

2 Log on using your usual RCP username and password

3 Select the relevant CME question paper

4 Answer all 10 questions by selecting the best answer from the options provided

5 Once you have answered all the questions, click on Submit

\section{Registering your external CPD credits}

Carrying out this activity allows you to claim two external CPD credits. These will be automatically transferred to your CPD diary, where you can review the activity and claim your points.

1 Which of the following is a risk factor for increased relapse rate in anti-neutrophil cytoplasmic antibody (ANCA)associated vasculitis?

(a) ANCA negative status after completion of induction therapy

(b) higher cumulative dose of cyclophosphamide

(c) higher serum creatinine at presentation

(d) microscopic polyangiitis

(e) proteinase 3 positive at presentation.

2 A 76-year-old man presented with painful unilateral proptosis. He had been feeling unwell for the previous few weeks with arthralgia, weight loss and cough. On close questioning, he admitted to nasal crusting and discharge. Examination revealed proptosis associated with lateral gaze diplopia. Computerised tomography scanning revealed a pseudo-tumour of the orbit, and proteinase 3 and ANCA is positive. He has normal renal function. Which of the following would be the most appropriate initial treatment?

(a) intravenous rituximab $1 \mathrm{~g}$ given now and another $1 \mathrm{~g}$ 2 weeks later

(b) methotrexate $20 \mathrm{mg}$ oral once per week

(c) plasma exchange

(d) pulsed cyclophosphamide 10-15 mg/kg given at 2-weekly intervals along with an oral glucocorticoid regimen

(e) pulsed intravenous methyl-prednisolone (500 mg to $1 \mathrm{~g}$ ) alone.

3 A 30 -year-old man with a long history of psoriasis presented with a swollen knee and painful fingers. His mother has rheumatoid arthritis. Which of the following features would be most helpful in the diagnosis of psoriatic arthritis?
(a) rheumatoid factor present in low titre
(b) psoriasis in scalp and natal cleft
(c) dactylitis of a finger
(d) large erosions of the metacarpal heads
(e) an elevated erythrocyte sedimentation rate.

4 Which of the following statements is true about the treatment of psoriatic arthritis?

(a) Methotrexate is of proven efficacy for skin and musculoskeletal manifestations.

(b) Biologic drugs are only indicated as first line treatment in the most severe cases.

(c) New targets for treatment include B-cells, IL-12 and IL-6.

(d) Oral steroids are recommended as first line treatment for psoriatic arthritis.

(e) Treatment has not demonstrated a beneficial effect on cardiovascular morbidity.

5 A 68-year-old lady presented with pain, swelling, and erythema of the first right metatarsophalangeal joint. This began abruptly overnight and the joint was exquisitely tender to touch. There was no history of injury or recent infections. She had a similar episode 2 years previously, which was diagnosed as acute gout, and was treated with colchicine $0.5 \mathrm{mg}$ twice a day for 1 week with complete resolution of symptoms. She had hypertension, angina, chronic kidney disease 3 (estimated glomerular filtration rate $55 \mathrm{~mL} / \mathrm{min}$ ) and mild cardiac failure. She is currently on losartan $50 \mathrm{mg}$ once daily, simvastatin $40 \mathrm{mg}$ once daily, aspirin $75 \mathrm{mg}$ once daily, furosemide $40 \mathrm{mg}$ once daily, and omeprazole $20 \mathrm{mg}$ once daily. 
What should be done about long-term pharmacologic urate-lowering therapy once the acute gout attack has subsided?

(a) Start febuxostat $80 \mathrm{mg} /$ day as this is more potent than allopurinol $300 \mathrm{mg} / \mathrm{day}$.

(b) Start low-dose allopurinol (100 mg/day) and up-titrate the dose according to serum uric acid.

(c) Start allopurinol (300 mg/day) and up-titrate the dose according to the serum uric acid level.

(d) Start vitamin C supplements and advise the patient to take cherry juice extract regularly to lower serum uric acid and prevent acute gout, respectively.

(e) Wait for an additional attack of acute gout to occur in the next 12 months before initiating pharmacologic urate lowering treatment.

6 A 58-year-old man presented with abrupt onset of right knee pain, swelling and stiffness to his local hospital accident and emergency (A\&E) department. He had a 5-year history of multiple self-limiting episodes of severe pain, swelling, and erythema affecting the first right metatarsophalangeal joint, the knee, the ankle or the elbow, and was diagnosed as having gout by his GP. A synovial fluid aspirate was performed in the $A \& E$, revealing negatively-birefringent needle-shaped crystals. The gram stain was negative and the 24 -hour culture was sterile. He currently takes losartan $50 \mathrm{mg}$ once daily, aspirin $75 \mathrm{mg}$ once daily, and allopurinol $500 \mathrm{mg} /$ day. The full blood count, urine and electrolytes and liver function tests were normal. The C-reactive protein was elevated at $124 \mathrm{mg} / \mathrm{dL}$ and the serum uric acid was $294 \mu \mathrm{mol} / \mathrm{L}$. The medical registrar diagnosed acute gout and performed an intraarticular corticosteroid injection.

\section{Which of the following statements best describes what should be done about allopurinol dose?}

(a) Continue allopurinol at the current dose during the acute gout attack and after the episode has subsided.

(b) Continue allopurinol at the current dose, recheck serum uric acid 2-3 weeks after the acute attack of gout, to determine whether the dose should be increased.

(c) Discontinue allopurinol for the duration of acute gout attack and restart at same dose after the episode has subsided.

(d) Increase allopurinol dose immediately as the patient is getting acute gout attacks despite being on allopurinol $500 \mathrm{mg} /$ day.

(e) Reduce allopurinol dose to $400 \mathrm{mg} /$ day as the serum uric acid is low.
7 What type of eye disease is seen most frequently in Behçet's syndrome?
(a) corneal ulceration
(b) episcleritis
(c) retinal vasculitis
(d) scleritis
(e) uveitis.

8 In the treatment of Behçet's syndrome, what drug takes the longest to demonstrate efficacy?
(a) azathioprine
(b) colchicine
(c) interferon-alpha
(d) systemic corticosteroids
(e) TNF inhibitors (such as infliximab).

9 Which of the following carries the greatest risk of developing systemic lupus erythematosus (SLE)?
(a) dizygotic twin with SLE
(b) first degree relative with SLE
(c) HLA B27
(d) HLADR4
(e) monozygotic twin with SLE.

10 A 22-year-old lady with a known history of systemic lupus erythematosus presented with severe sharp chest pain. It was worse on lying flat. She was apyrexial with a pulse rate of $75 \mathrm{bpm}$ and blood pressure of $110 / 78 \mathrm{mmHg}$. Her heart sounds and breath sounds were normal on auscultation. Her electrocardiogram showed sinus rhythm with widespread ST elevation. Her troponin was normal. What is the most likely diagnosis?
(a) autoimmune pericarditis
(b) Libman-Sacks endocarditis
(c) myocarditis
(d) ST elevation myocardial infarction
(e) viral pericarditis..

\section{CME Immunology and allergy SAQ Answers to the CME SAQ published in Clinical Medicine in December 2016}
Q1 Q2 Q3
Q4 Q5
Q6
Q7 Q8 Q
Q10
(b) (a) (d)
(d) (d)
(a) (d) 